\title{
Three-Dimensional Characterization of Oxide Fuel Utilizing Focused Ion Beam Tomography
}

\author{
Casey McKinney and Assel Aitkaliyeva
}

University of Florida, United States

Scanning electron microscopy (SEM), electron backscatter diffraction (EBSD), and energy dispersive x-ray spectroscopy (EDS) are commonly used to characterize structural and chemical features in many materials. The inherent nature of the techniques limits them to two-dimensional (2D) surface analysis, but the incorporation of these with focused ion beam (FIB) tomography can add on the third dimension. FIB tomography is process that involves collecting an image or scan of the surface of a pre-made volume, then milling away a specified amount of material, then collecting an image or scan of the newly visible surface. This process is sequentially repeated until the desired volume of material has been characterized. Then with some post-processing image analysis, the 3D structure of the material can be reconstructed. Utilizing this technique to section volumes of oxide fuel can provide detailed 3D microstructural and chemical reconstructions for different regions of the fuel.

During reactor operation, harsh environments and steep temperature gradients cause both structural and compositional changes in nuclear fuel. For example, restructuring in light water reactor (LWR) $\mathrm{UO}_{2}$ results in grain sizes differing by a factor of 10 in regions less than half a millimeter apart [1]. The fission, or splitting of atoms, produces new phases and precipitates that have different, possibly undesirable, properties compared to the asfabricated state of the fuel [2]. As grain size and chemical composition affect the mechanical, thermal, and transport properties that were designed for safe reactor operation, it is important to understand their evolution over their lifetime in the reactor to avoid situations that might result in fuel failure. Just as the solid fission products have the potential to negatively impact fuel performance, gaseous fission products do as well. When volatile fission products coalesce and migrate, interconnected bubble networks can form resulting in significant fission gas release [3]. This gas release can lead to cladding burst, which would release these volatile fission products into the reactor and compromise safety [4]. Understanding the evolution of bubbles, grains, and fission products is a key factor in ensuring safe reactor operation. As these features exist in three-dimensions (3D), especially interconnected bubble networks, they should therefore be characterized in 3D to prevent missing any critical anisotropic facets of their structure.

To fill the gap in current understanding of the fuel performance we characterize blocks of oxide fuels using FIB tomography. This allows us to assess the state of the bubble structure and fission products in different regions. Previous investigation of a sample of mixed oxide (MOX) fuel showed Pd/Te precipitates on open bubble surfaces, shown in Figure 1 [5]. To investigate the 3D microstructural nature of these features, we employed FIB tomography with simultaneous EBSD/EDS. Figure 2 shows the resulting five metal precipitate (FMP) and Pd/Te precipitate reconstruction. The largest FMPs formed on open bubble surfaces, which was confirmed with the SEM micrographs. These large FMPs on open bubble surfaces also served as nucleation sites for the Pd/Te precipitates, which was expected from the previous nanoscale investigation. These precipitates were postulated to have formed due to volatile Pd and Te diffusing away from the hot central region of the fuel and nucleating on FMPs on open bubbles surfaces in cooler regions of the fuel [5]. 3D characterization provided the means to properly characterize the structure of the $\mathrm{Pd} / \mathrm{Te}$ precipitates on the anisotropic bubble in the region, which uncovered that the $\mathrm{Pd} / \mathrm{Te}$ precipitates were larger than expected from initial surface characterization. 


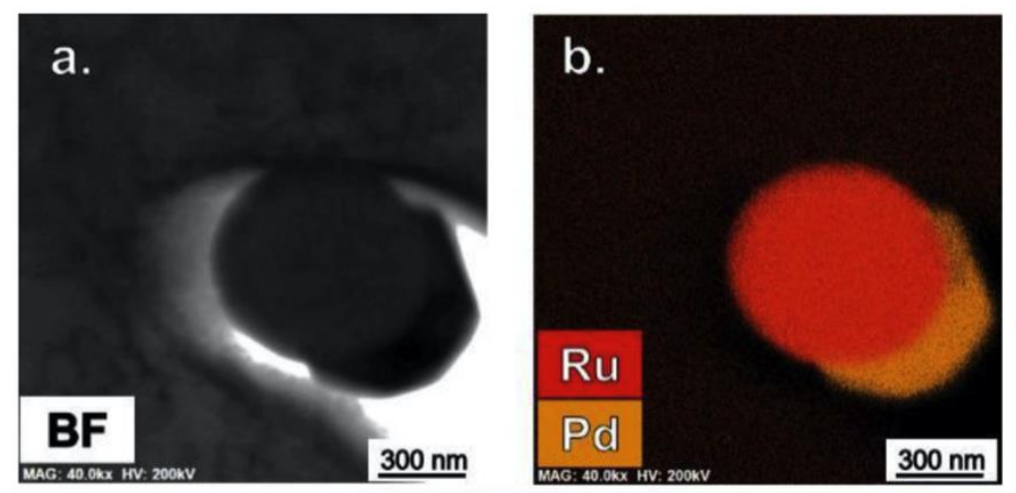

Figure 1. a.) Scanning transmission electron microscope (STEM) bright field image of FMP and Pd/Te precipitate on an open pore. b.) STEM-EDS map showing Pd and Ru distribution.

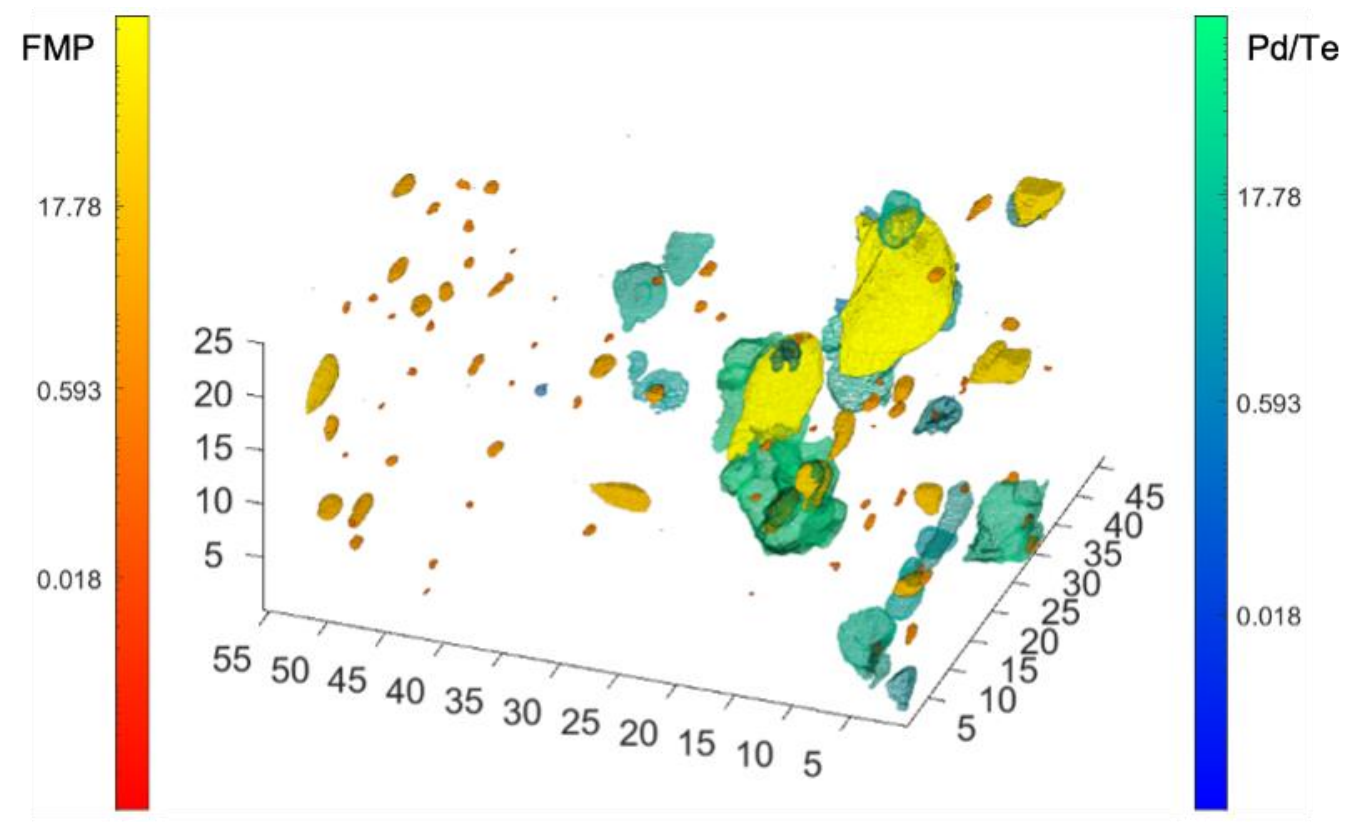

Figure 2. FMP and Pd/Te precipitate reconstruction from a MOX fuel pellet with feature size color coded by relative volume $(\mu \mathrm{m} 3)$ and axes scaled in microns.

\section{References}

[1] T. Gerczak et al., Journal of Nuclear Materials 509 (2018), p. 245-259.

[2] H. Kleykamp, Journal of Nuclear Materials 131 (1985), p. 221-246.

[3] W. Beere and G. Reynolds, Journal of Nuclear Materials 47 (1973), p. 51-57.

[4] T. Fuketa et al., Journal of Nuclear Materials 248 (1997), p. 249-256.

[5] R. Parrish et al., Journal of Nuclear Materials 524 (2019), p. 67-79. 